\title{
Los enterramientos neolíticos de Ca l'Arnella (Terrassa, Barcelona)
}

\author{
The Neolithic burials of Ca l'Arnella (Terrassa, Barcelona)
}

\author{
Roser Pou (*) \\ Miquel Martí (*) \\ Millán Mozota $(* *)$
}

\author{
Núria Armentano (***) \\ Patricia Martín (****) \\ Juan F. Gibaja (**)
}

\section{RESUMEN}

La prospección e intervención arqueológica en el yacimiento de Ca l'Arnella motivadas por las obras del gaseoducto Martorell-Figueres propiciaron la localización y excavación de dos hipogeos funerarios con inhumaciones individuales del Neolítico medio (finales del V milenio e inicios del IV milenio cal AC). Las estructuras, inhumados y materiales documentados son estudiados en este trabajo. Un geométrico hallado junto a las vértebras lumbares de uno de los inhumados pudo estar alojado en el interior del cuerpo y muestra estigmas típicos de su uso como proyectil, por lo que tal vez estemos ante una de las pocas evidencias de violencia durante este periodo. El análisis de los datos arqueológicos contextuales aportó varias líneas de evidencia que apoyan esta posibilidad.

\section{ABSTRACT}

The archaeological survey and intervention on the $\mathrm{Ca}$ l'Arnella site were motivated by the construction works

(*) Associació de Tècnics d'Investigacions Culturals i Socials, s.l (ATICS SL). C/ Torrent de les Piques 38 (baixos). 08304 Mataró. España.

Correos e.: roser@gmail.com; miqros@gmail.com

$(* *)$ Institució Milà i Fontanals (IMF-CSIC). C/Egipcíaques 15. 08001 Barcelona. España.

Correos e.: millanm@imf.csic.es; jfgibaja@imf.csic.es

$(* * *)$ ANTROPOLEGS.LAB Y GROB (Grup de Recerca en Osteobiografía) de la Universidad Autónoma de Barcelona C/ Rossend Arús 46, baixos 1A. 08014 Barcelona. España.

Correo e.: armentano.nuria@gmail.com

(****) Institut de Paleoecologia Humana i Evolució Social (IPHES). C/ Marcel-li Domingo s/n. Campus Sescelades (Edifici W33). 43007 Tarragona. España. Correo e: patrimr9@gmail.com Recibido 5-III-2013; aceptado 9-V-2013. of the Martorell-Figueres pipeline, and led to the finding and excavation of two funerary hypogeal with individual burials from the Middle Neolithic. The structures, buried individuals and materials are studied in this article. A geometric flint implement found near the lumbar vertebrae may have been originally inside the body and shows typical stigma of projectile use. This could be one of the scarce pieces of evidences for violence during this peri$o d$. The analysis of archaeological data provided several lines of evidence supporting this possibility.

Key words: Prehistoria reciente; Neolítico medio; Arqueología de la muerte; Huellas de uso; Antropología física; Noreste de la Península Ibérica; Proyectil; Violencia; Sepulcros de fosa; Mediterráneo.

Palabras clave: Recent prehistory; Middle Neolithic; Archaeology of Death; Use-wear analysis; Physical Anthropolgy; Northeast of Iberian Peninsula; Projectile; Violence; Pit graves; Mediterranean.

\section{INTRODUCCIÓN Y DESCRIPCIÓN DEL YACIMIENTO}

La prospección, control y seguimiento arqueológico de la obra del gasoducto Martorell-Figueres pusieron al descubierto diversas evidencias en el tramo comprendido entre Martorell y Hostalric. En el término municipal de Terrassa, el gasoducto afectaba directamente a dos yacimientos ya conocidos: Camps de Can Colomer (parcialmente excavado durante las obras de la infraestructura viaria del 4art cinturó) y Ca l'Arnella 
(Fig. 1) con materiales de época romana conocidos en los 1960 (1).
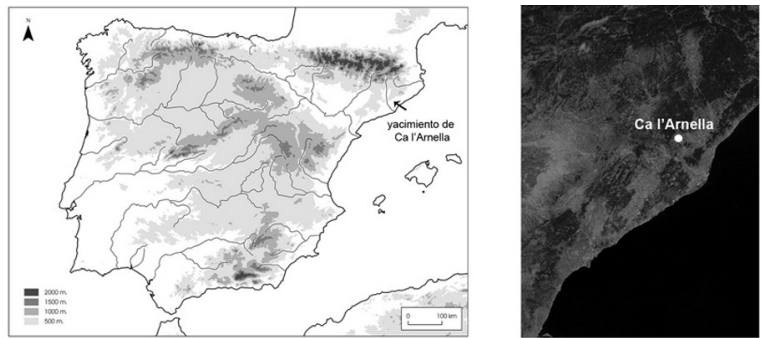

Fig. 1. Localización del yacimiento de Ca l'Arnella. A la derecha, detalle de la región circundante en la actualidad (incluyendo el Vallès, el Barcelonés y el Maresme); Base cartográfica BCN IGM.

Ca 1'Arnella se sitúa entre el torrente de la Batzuca y la carretera BV-1248 en el límite con el término municipal de Sabadell (comarca del Vallès Occidental). Es un llano con ligera pendiente hacia el sur que conserva su función agrícola. El yacimiento, alzado a 355 m.s.n.m., se asienta sobre un manto aluvial del Pleistoceno formado por arcillas carbonatadas, limos y gravas.

Se conocen muchos contextos en esta comarca de la Prehistoria reciente, ya desde el Neolítico antiguo. Ello se debe básicamente a las óptimas condiciones del territorio para la implantación y desarrollo de las prácticas agropecuarias. El asentamiento de Can Roqueta (Sabadell) con una ocupación casi continuada desde el Neolítico hasta época medieval (González et al. 1999) es ejemplo de ello.

Las prospecciones, dirigidas por la empresa ATICS S.L. entre diciembre del 2011 y enero del 2012 para evaluar el impacto ambiental tanto del 4art cinturó como del gasoducto, tuvieron por objetivo delimitar los yacimientos arqueológicos. Se documentaron 12 estructuras negativas: 5 fondos de silo (uno de ellos de época romana y el resto de cronología indeterminada), 3 fosas ovaladas prehistóricas (posiblemente neolíticas) cuya función desconocemos, 1 estrato de arcillas con material cerámico de la Edad del Bronce Inicial, 1 zanja de viña subactual y 2 tumbas hipogeas

(1) En el Catàlogo de l'Inventari del Patrimoni Arqueològic de Catalunya. http://cultura.gencat.cat/invarque/index.asp (consulta 15-V-2013). del Neolítico medio, situadas a escasa distancia una de otra, que estudiamos en el presente artículo.

\section{LOS HIPOGEOS NEOLÍTICOS: CONSTRUCCIÓN Y DISPOSICIÓN DE LOS INHUMADOS}

Las dos tumbas hipogeas, CCA-2 y CCA-3, corresponden al tipo $5 \mathrm{~b}$, según tipología de Pou et al. (1994), Pou y Martí (1997). Son estructuras complejas con pozo de acceso y cámara lateral.

El pozo de acceso del hipogeo CCA-2 tenía una banqueta inicial de planta cuadrangular y estaba colmatado de piedras. La cámara lateral absidial había sido excavada unos $45 \mathrm{~cm}$ por debajo de la base del pozo (Fig. 2: 1). Se orienta Noroeste-Sureste y tiene forma circular $(1,85 \mathrm{~m}$ de diámetro). La bóveda de dicha cámara había cedido. Bajo sus escombros un estrato de arcilla marrón más suelta cubría los restos esqueléticos, localizados en la base de la cámara.

Se halló un individuo articulado en decúbito supino, orientado NO-SE, con el cráneo a SE (Fig. 2: 2). Presentaba flexión de codo izquierdo, antebrazo cruzando la zona torácica-abdominal y elementos de la mano desarticulados por encima del coxal derecho y sacro. También mostraba extensión de las extremidades inferiores, con una lateralización izquierda de ambos pies.

El cráneo, la mandíbula y el húmero derecho se situaban cerca de la pared opuesta al acceso. A excepción de esos tres elementos desplazados, la forma de las articulaciones anatómicas era estricta (hombro izquierdo, coxofemorales, rodillas, tobillos) o ligeramente laxa (muñeca izquierda, sacroilíacas, peroneotibial superior izquierda), sugiriendo una colmatación bastante rápida de la sepultura, previa a la total descomposición de los tejidos blandos (Ubelaker 1989; Duday et al. 1990).

La posición del cráneo, mandíbula y húmero a una cota superior a la del resto del esqueleto hace improbable un rodamiento por gravedad. Esos elementos podrían haber sido movidos de forma voluntaria cuando se encontraban en fase esquelética, ya que no hay indicios de desmembramiento intencional ni marcas en las superficies peri-articulares afectadas. Es interesante remarcar que, según los estudios sobre la secuencia de desarticulación y los procesos conexos, cráneo y 
Sepultura CCA-2
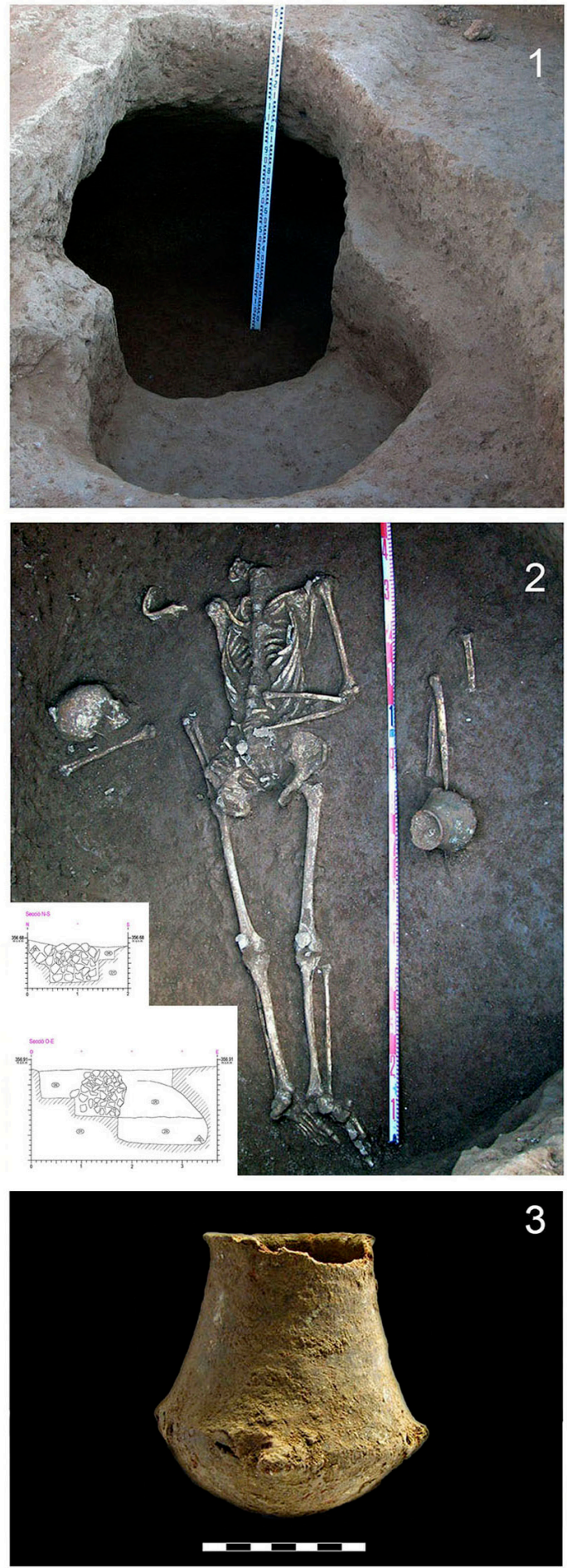
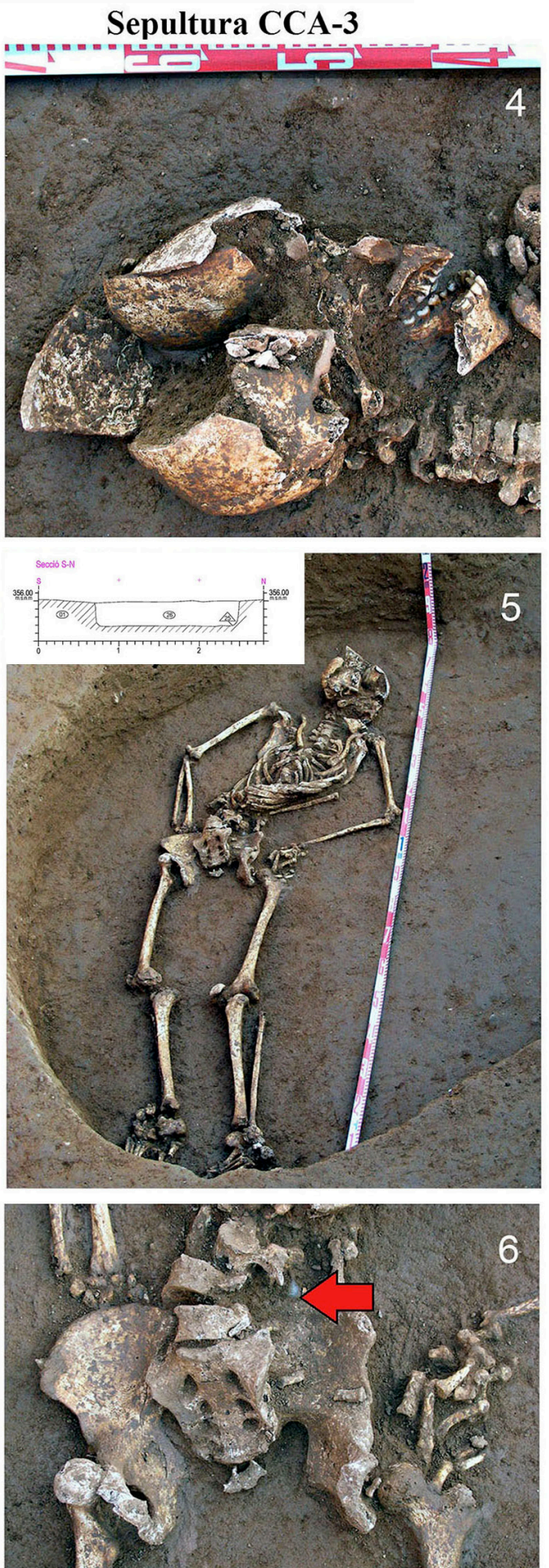

Fig. 2. Hipogeos de Ca l'Arnella (Terrassa, Barcelona). Neolítico medio/fines del V milenio - inicios del IV milenio cal AC. CCA-2: 1. entrada a la cámara; 2. disposición de la mujer con ajuar a su izquierda y secciones de la sepultura; 3. vaso globular con cuatro aplicaciones. CCA-3: 4. cráneo del varón; 5. disposición del esqueleto y sección de la sepultura; 6. la flecha en la zona coxal localiza la posición del geométrico de sílex. 
mandíbula, debido al tipo de articulación y a su relativa cantidad de tejido blando, son los primeros elementos que pierden su conexión articular. A su vez las extremidades superiores preceden a las inferiores (Lyman 1994; DiMaio y DiMaio 2001; Haglund y Sorg 2002). El cráneo pudo estar, en origen, a una cota más alta, si la cabeza descansaba sobre un apoyo. Precisamente, allí se localizó un sedimento más suelto, con numerosos carbones (de los que se recogieron muestras), que podría demostrar la preparación de esta zona con materias vegetales. El estado de preservación esquelética es del $100 \%$, a pesar de que existen alteraciones tafonómicas en la cortical ósea que afectan de forma importante a los elementos de cintura escapular y pélvica (Armentano et al. 2012).

El ajuar funerario se localizó al Oeste de la cámara, justo delante de la entrada. Constaba de dos punzones de hueso, un radio de ovicaprino no trabajado, una plaqueta de variscita y un vaso cerámico. En el pozo de acceso, en el nivel de colmatación de piedras, había restos del cráneo de un cánido.

El hipogeo CCA-3 tenía un pequeño pozo de acceso y cámara lateral (de 1,90 m de largo por 1,50 m de ancho) orientada NO-SE. El pozo y la bóveda de la cámara habían cedido y se encontraban rellenas de piedras.

En su interior había un individuo adulto, articulado en decúbito supino, orientado NO-SE, con el cráneo al SE. Se observó lateralización izquierda de cráneo, abducción de extremidades superiores, semi-flexión de codos, manos al nivel de la cintura (pronación de la derecha y desarticulación de los elementos de la izquierda). También presentaba extensión de las extremidades inferiores. La mitad superior del cuerpo tenía una ligera lateralización izquierda y la mitad inferior lateralización derecha. Todas las articulaciones anatómicas se mantuvieron de forma muy laxa, sugiriendo una descomposición de los tejidos blandos con la sepultura en espacio vacío. Es coherente con ello la diástasis sacro-ilíaca bilateral, la desarticulación bilateral de la cabeza del fémur y las desarticulaciones de las rodillas y de algunos elementos de manos y pies (Ubelaker 1989; Duday et al. 1990).

El hecho de haber hallado las clavículas paralelas y siguiendo el eje longitudinal del cuerpo puede sugerir que el individuo se enterró con los hombros encogidos. El estado de preservación esquelética es del 100\% (Armentano et al. 2012), a pesar de que la conservación del material es mediana por alteraciones tafonómicas generalizadas (fisuras, irregularidades y rugosidades la cortical ósea). Presenta fracturas post-mortem a nivel craneal y una importante degradación de los elementos del esplacnocraneo. Se observó una posición forzada de la parte inferior de hemitórax izquierdo: las costillas izquierdas 8-11 mantenían una posición desplazada hacia la derecha y por encima de los cuerpos vertebrales. Además la desarticulación de las últimas vértebras dorsales y las dos primeras lumbares era importante. La entrada de bloques en la cámara tras el hundimiento de la cámara y del pozo de acceso pudo desplazar algunas partes del individuo.

En el interior de la sepultura solo se halló un trapecio de sílex situado entre la tercera vértebra lumbar y el margen superior del ala ilíaca izquierda, junto a la piedra que presiona las costillas (Fig. 2: 6).

\section{ANÁLISIS ANTROPOLÓGICO}

Los restos corresponden a dos individuos de edad adulta de sexo femenino (CCA-2) y masculino (CCA-3). El estudio de la determinación de edad esquelética indica que los dos murieron con más de 30 años, siendo la mujer un poco mayor que el varón (Lovejoy et al. 1985; Krogman e Iscan 1986; Buikstra y Ubelaker 1994). Las alteraciones tafonómicas de las superficies auricular y sínfisis púbica de coxal y de los extremos costales no han permitido precisar más la edad.

Los huesos de ambos individuos en general son gráciles. Debido a una marcada remodelación diafisaria los huesos largos presentan aplanamientos importantes (platibraquia, platimeria y platicnemia). La talla de la mujer, estimada a partir de las fórmulas de regresión de Pearson sobre los datos antropométricos del fémur y el húmero, es de 153,55 cm (mesosoma). La talla calculada del varón es 157,27 cm (camesoma) (Martin y Saller 1957; Olivier 1960).

La mujer tiene un cráneo alargado en norma superior, con un importante número de huesos wormianos. Las suturas craneales se presentan libres. A nivel postcraneal se observa la apertura septal bilateral en húmeros, y carillas accesorias en la epífisis distal de tibias (Brothwell 1987). El varón destaca por la morfología robusta de la 
mandíbula, con un margen inferior grueso, y un mentón grande y prominente (Olivier 1960).

Como patología ambos individuos muestran una calcificación de los ligamentos amarillos en las últimas vértebras dorsales, y corona osteofítica en los cuerpos dorsales y lumbares. La mujer presenta una lesión lítica en el tercio proximal de húmero, a nivel del surco bicipital, y exóstosis entesopáticas bilaterales al margen superior de la cara anterior de las rótulas. En el varón las exóstosis están en la cara posterior de los calcáneos. En los dos esqueletos se han observado lesiones de etiología microtraumática, redondeadas, bien definidas de entre 4 y $8 \mathrm{~mm}$, compatibles con las habituales osteocondritis. En el varón esta lesión aparece a nivel de la epífisis distal del radio izquierdo, y en la mujer a nivel del radio derecho (Isidro y Malgosa 2003).

En ambos un desgaste dentario avanzado va acompañado de retroceso alveolar generalizado (Chimenos et. al 1999). La mujer tiene cúmulos de sarro y una fístula (a nivel del primer molar superior derecho). El varón se caracteriza por una malposición dental mandibular de las piezas anteriores derechas, una caries (a nivel del primer molar superior derecho) y un desgaste desigual de los primeros molares respecto a los posteriores, siendo el desgaste de las primeras piezas molares mucho mayor que el resto de piezas dentales.

\section{OBJETOS E INSTRUMENTOS DOCUMENTADOS}

En los estratos de colmatación del pozo y la cámara del hipogeo CCA-2 se recuperaron algunos fragmentos cerámicos informes $\mathrm{y}$, como parte del ajuar, un vaso de cuello diferenciado, cuerpo globular y cuatro pequeñas aplicaciones diametralmente opuestas situadas en la inflexión del cuello y el cuerpo. Mide 14,2 cm de altura, $8,2 \mathrm{~cm}$ de diámetro y el grosor de las paredes oscila entre 0,4 y $0,8 \mathrm{~cm}$. La cocción es neutra con predominio de la oxidante. El acabado interior y exterior es alisado, configurando un buen acabado. Las paredes del cuello son ligeramente cóncavas y el labio recto. Estos vasos son habituales en sepulturas contemporáneas de necrópolis vecinas como Camí de Can Grau (Granollers, Barcelona) y Can Gambús (Sabadell, Barcelona) (Pou et al. 1994; Roig et al. 2010). A falta de estudios sobre la composición de las pastas, se ha considerado que fueron producidos bajo la influencia de tradiciones técnicas típicas del Chassey francés. En Camí de Can Grau se relacionan exclusivamente con el tipo constructivo $5 \mathrm{~b}$, fechado en torno a 3600-3500 cal AC (2), el más reciente de las morfologías funerarias en fosa del Neolítico medio. Los recipientes contendrían la ofrenda propiamente dicha, algún tipo de bebida utilizada en el ritual funerario.

También se ha hallado una plaquita de variscita de $12 \times 6 \times 4 \mathrm{~mm}$ de forma ovalada y sin perforación. Parece la sección de una cuenta de collar tuneliforme, pero no observamos los rastros de su típica perforación longitudinal. Se localizaba entre el radio de ovicaprino y dos punzones de hueso de los que vamos a hablar a continuación.

El radio, derecho y entero, es de un ovicaprino juvenil, sin evidencias de procesado antrópico, ya sea con fines productivos o de consumir el animal. La edad, entre 12 y 24 meses, se estimó a partir del grado de fusión ósea de las epífisis: en la proximal es completa y la distal aún está por fusionar. Si bien no es posible precisar con seguridad si es de oveja o cabra, por la gran similitud morfológica de ambas especies, podría ser de oveja considerando su tuberosidad bicipital con tendencia redondeada.

Los dos punzones óseos conservan la mitad de la epífisis distal. Han sido estudiados con un enfoque tafonómico, tecnológico y de huellas de uso (Buc 2001; van Gijn 2005). Para ello, hemos combinado la observación macroscópica (x5-x80 aumentos) y microscópica (x100-x400).

El primer punzón es un metápodo de un bóvido adulto con una longitud de $279 \mathrm{~mm}$, una anchura en su bisectriz transversal de $18 \mathrm{~mm}$ y un espesor en el mismo punto de $8 \mathrm{~mm}$ (Fig. 3). La sección es de tendencia cuadrangular aplanada en la zona distal, y de tendencia ovalada y aplanada en la proximal. En superficie hay alteraciones producto del agua filtrada y de la acción de las raíces.

Presenta dos ranuras tecnológicas. Una en la parte proximal derecha tiene 1-1,5 mm de anchura y unos $10 \mathrm{~cm}$ de longitud y en su interior se observan estrías longitudinales largas y anchas

(2) Dataciones realizadas en la necrópolis de Camí de Can Grau publicadas por Martín (2009: 57).

Trab. prehist., 71, N. ${ }^{\circ} 1$, enero-junio 2014, pp. 146-155, ISSN: 0082-5638

doi: $10.3989 /$ tp.2014.12129 


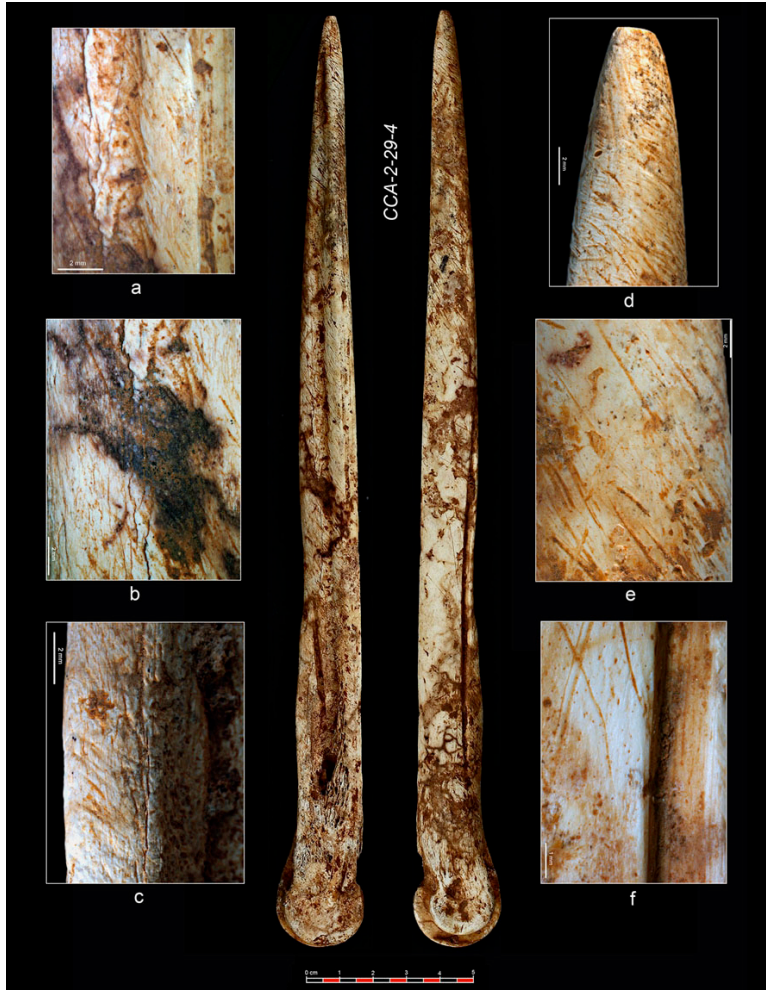

Fig. 3. Ca l'Arnella (Terrassa, Barcelona). Punzón óseo CCA-2-29-4: vistas completas, cortical (dorsal) y medular (ventral), en el centro; a. estrías oblicuas (vista medular) y longitudinales (en bisel lateral) de la zona distal; b. detalle de alteración erosiva asociada a las marcas de raíces; c. estrías oblicuas (vista medular, lateral izquierdo) de la zona proximal del soporte; d. concentración de estrías oblicuas en zona distal cercana al ápice (vista cortical); e. estrías oblicuas en la zona mesial (vista cortical); f. ranura longitudinal y estrías en el interior (zona proximal, vista cortical).

(Fig. 3: f). La otra, en la parte medular, es de unos $4 \mathrm{~cm}$ de longitud e idéntica anchura.

Las estrías generadas durante el proceso de fabricación cubren la superficie. Unas son longitudinales y largas (más de $2 \mathrm{~cm}$ ), paralelas entre sí y agrupadas en haces. Otras son más cortas (menores de $1 \mathrm{~cm}$ ) y dispuestas oblicuamente al eje del útil. Unas y otras se generaron de manera sucesiva con instrumentos líticos: útiles tallados para raspar o yunques para abrasión. Junto a estas estrías de origen tecnológico, otras muy finas, longitudinales y transversales, próximas al ápice de la pieza, pudieron formarse al perforar con el punzón. Sus peculiaridades no nos permiten definir la materia trabajada.

El segundo punzón, elaborado a partir de un metápodo de ungulado de talla pequeña, mide
$174 \mathrm{~mm}$ de longitud, $14 \mathrm{~mm}$ de anchura y $5 \mathrm{~mm}$ de espesor. La sección es de tendencia cuadrangular, aplanada en la zona distal y semicircular en la proximal. Ha sufrido igualmente alteraciones tafonómicas atribuibles a la acción disolvente de la humedad y los agentes químicos que transporta el agua de los sedimentos.

Entre los estigmas de fabricación advertimos un ligero adelgazamiento del ápice y un conjunto de estrías de varios tamaños y direcciones, localizadas en distintas zonas del punzón. Un grupo de estrías oblicuas y cortas se concentran en el ápice y en los biseles de fractura de la zona cercana a la epífisis, en la cara medular. El redondeamiento de su topografía disminuye a medida que nos alejamos del ápice, lo que puede vincularse con su utilización. Gracias a la observación microscópica pudimos asociarlo a otras modificaciones producto del uso. Un pulido concentrado en las zonas altas asociado a estrías de diverso tamaño, transversales y longitudinales al eje del soporte (Fig. 4), sugiere su empleo para perforar una material animal, como una piel.

Como recordaremos, el único instrumento hallado en el hipogeo CCA-3 es un geométrico de sílex de morfología aparentemente triangular. Tenemos ciertas dudas de que fuera la original por las intensas fracturas que ha sufrido buena parte de su perímetro. Su localización en la zona lumbar del varón nos llevó a pensar que pudiera proceder del interior del cuerpo. Para determinarlo realizamos, junto a la Policía Cientifica dels Mossos d'Esquadra, un análisis dirigido a la detección de hemoglobina. Este tipo de análisis está en una fase muy experimental, ya que por el momento desconocemos las condiciones y duración de conservación de la hemoglobina. Era una oportunidad excepcional, ya que el geométrico había sido guardado en una bolsa individual y sin limpiar y la limpieza con agua o detergente habría eliminado los restos de sangre.

Empleamos un kit Hexagon OBTI, que permite reconocer mediante la reacción de determinadas proteínas la existencia de restos de hemoglobina de primates (Hochmeister et al. 1999). Desconocemos por qué los resultados fueron negativos. Si el geométrico efectivamente estuvo en el cuerpo, el tiempo transcurrido, el tipo de sedimento o la humedad pudieron facilitar la desaparición de la sangre.

Para aproximarnos a la función de la pieza estudiamos a escala macro y micro su superficie. 


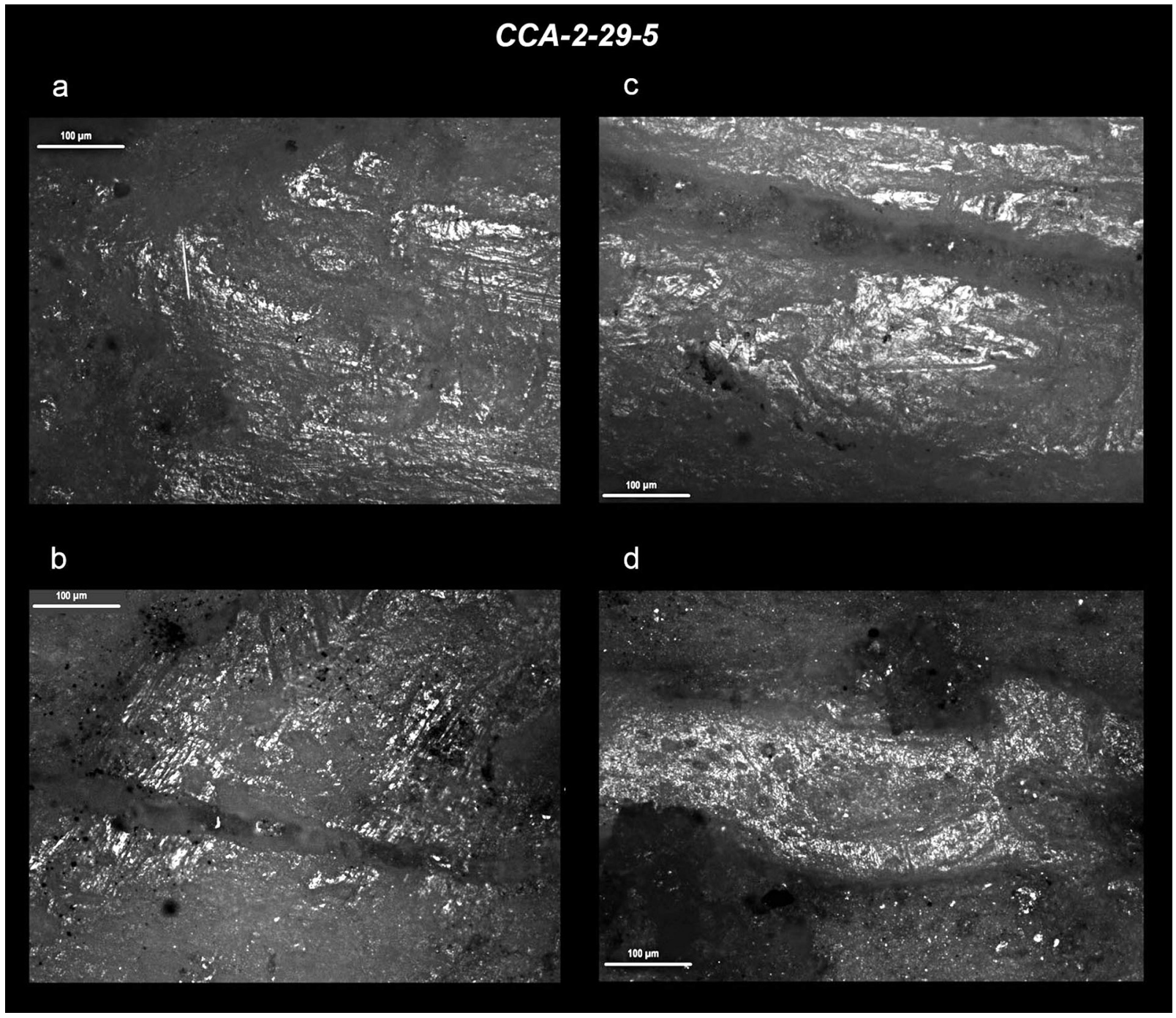

Fig. 4. Ca l'Arnella (Terrassa, Barcelona). Estigmas microscópicos a x200 aumentos en punzón óseo CCA-2-29-5 en regiones próximas al ápice o extremo distal (a. vista cortical a $2 \mathrm{~mm}$ del ápice; b. vista medular a $20 \mathrm{~mm}$ del ápice; c. bisel lateral a $10 \mathrm{~mm}$ del ápice) y en zona proximal (d. vista cortical).

Adquirimos una ínfima información del análisis microscópico por el intenso lustre de suelo del sílex. Este hecho nos impidió detectar modificaciones apreciables a altos aumentos $(>100 \mathrm{X})$ como estrías y micropulidos.

En la observación macroscópica identificamos fracturas en la parte apical y en el filo corto sin retocar, resultantes del uso del geométrico como proyectil. Este tipo de fracturas solo se genera cuando el sílex entra en contacto de manera violenta con una materia muy dura, como un hueso (Gibaja y Palomo 2004). Hemos observado una fractura de morfología aburilada en uno de los vértices, entre el filo largo no retocado y uno de los laterales retocados. Esta fractura ha suprimido gran parte del filo retocado (Fig. 5: 1). Con esta fractura apical se asocia otra también aburilada que nace del filo corto. Posiblemente es resultado del fuerte contragolpe contra el astil. Estas roturas son muy habituales cuando el golpe es muy intenso (Fig. 5: 2). La morfología de algunas melladuras en el filo largo sin retocar tiende a orientarse en diagonal al filo. Esa direccionalidad nos permite suponer que el eje de la pieza no era paralelo al del astil si no ligeramente diagonal (Fig. 5: 3). Desconocemos el origen del residuo

Trab. prehist., 71, N. ${ }^{\circ}$ 1, enero-junio 2014, pp. 146-155, ISSN: 0082-5638

doi: $10.3989 /$ tp.2014.12129 


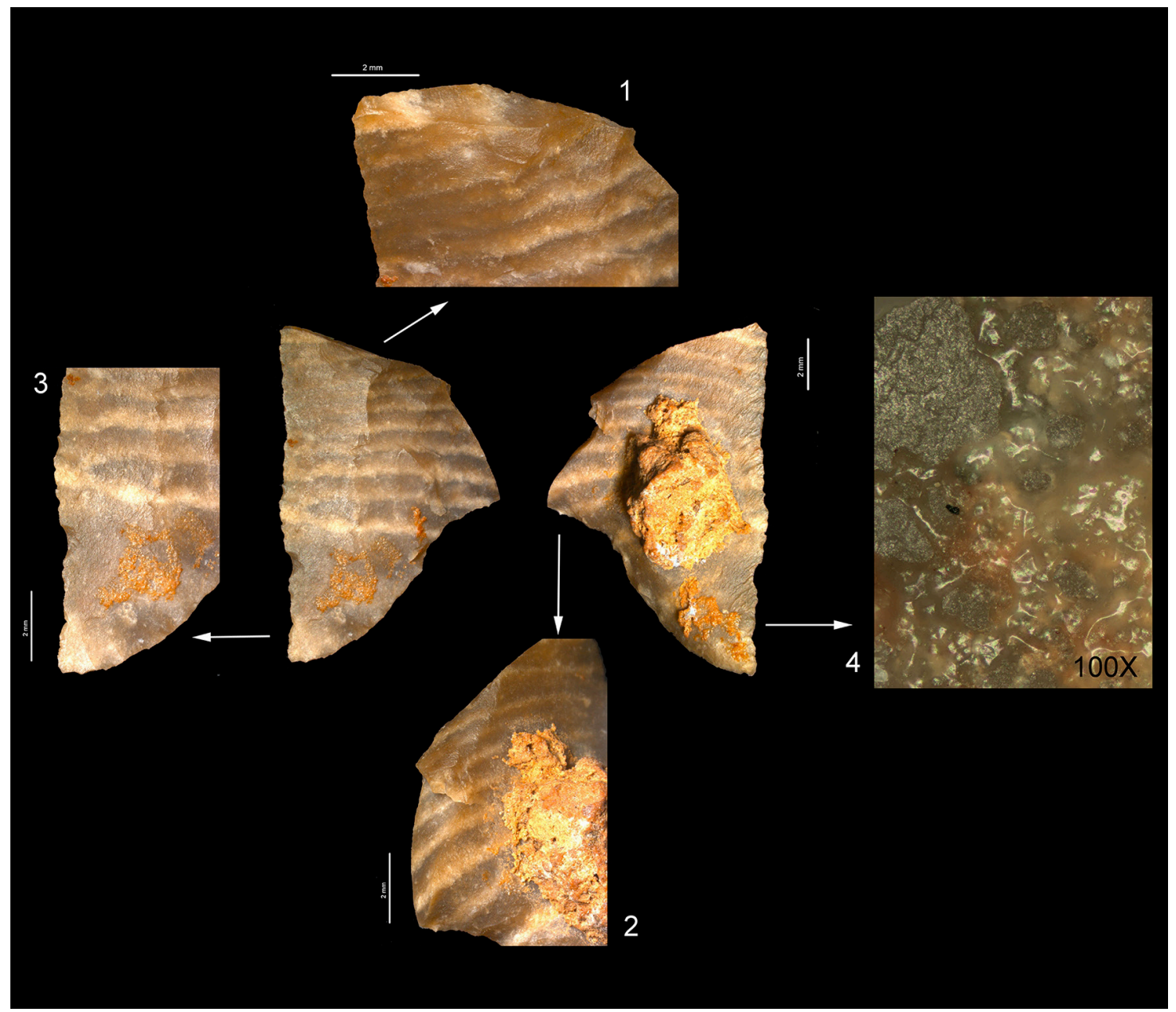

Fig. 5. Ca l'Arnella (Terrassa, Barcelona). Neolítico medio/fines del V milenio - inicios del IV milenio cal AC. Geométrico de sílex documentado en la zona coxal del varón inhumado en el hipogeo CCA-3: fracturas aburiladas 1. en ápice y 2. en filo corto; 3. melladuras en filo largo sin retocar; 4. posibles residuos de mastique.

del ápice contrario al fracturado (Fig. 5: 4) cuya naturaleza merecería un futuro análisis. En otros yacimientos neolíticos del noreste peninsular (Gibaja y Palomo 2004), hemos documentado muchos geométricos con residuos negruzcos pertenecientes al mastique empleado para enmangarlos.

Este conjunto de modificaciones nos permiten afirmar que el geométrico fue empleado como proyectil y enmangado probablemente como punta o barbelure.

\section{CONCLUSIONES}

Cuando hablamos del Neolítico medio en Cataluña (finales del V milenio e inicios del IV cal
AC) es obligado remitirnos a los contextos funerarios. Durante este periodo, el registro arqueológico está caracterizado por sepulturas y silos/fosas de desecho. Sólo se conocen estructuras de hábitat en el complejo minero de Gavà (Barcelona), donde también se han hallado inhumaciones.

Ca l'Arnella aporta dos hipogeos excepcionales, CCA-2 y CCA-3, que conservan toda la estructura y se han excavado de manera cuidadosa con métodos actuales. Ello nos ha permitido determinar que son sepulturas "monumentales" del tipo 5b de Pou et al. (1994) y Pou y Martí (1997). Uno de los aspectos más singulares es la localización de un geométrico de sílex cerca del coxal izquierdo y de las vértebras lumbares del varón del hipogeo CCA-3. Aunque no se observan sig- 
nos traumáticos en la superficie del coxal o de las vértebras lumbares, el geométrico presenta significativas fracturas de impacto producidas por su uso como elemento de proyectil. Para saber si tales fracturas reflejan el alojamiento del geométrico en el individuo hay que evaluar ciertos aspectos.

La ausencia de traumatismos en los restos óseos debe tener en cuenta que la conservación de los mismos no es excelente. El geométrico podría haber impactado en la periferia de algún hueso, sin que tal lesión fuera detectable en la actualidad.

Las claras fracturas de impacto en el geométrico no suelen constatarse en otros contextos funerarios contemporáneos. Por lo general, los geométricos se depositaron sin usar o con un uso que solo implicó pequeñas roturas. Las comunidades neolíticas no solían incorporar al ajuar proyectiles fracturados.

Lo común no es encontrar un solo microlito como en CCA-3 si no varios asociados, a veces incluso con otros proyectiles como las puntas. Menos habitual todavía es que un geométrico sea la única pieza hallada en una tumba. Por ejemplo, hemos estudiado tres de las mayores necrópolis neolíticas catalanas: Bòbila Madurell (67 tumbas, 12 de las cuales tienen geométricos), el Camí de Can Grau (25 sepulturas, 9 con geométricos) y Can Gambús 1 (47 sepulcros, 16 con geométricos) y en los enterramientos con geométrico este siempre se asocia con otros elementos de ajuar.

El varón de CCA-3, a pesar de estar en decúbito supino extendido, sugiere una disposición original ambigua, por la colocación de las costillas izquierdas y lumbares, de las extremidades inferiores y de la cintura pélvica respecto la parte superior del cuerpo. No está asociado a ningún ajuar. Podemos encontrar en otros yacimientos contemporáneos tumbas sin ajuar, pero ello sucede con mayor asiduidad en los enterramientos parcial o totalmente destruidos.

Por último, la localización de un geométrico aislado entre la tercera vértebra lumbar y el margen superior del ala ilíaca izquierda es un dato altamente relevante. Lo habitual (Gibaja 2003) es encontrar depositados los geométricos en los laterales del inhumado y, en ocasiones, agrupados en tanto que formaban parte de un conjunto de flechas o estaban almacenados en algún tipo de recipiente, desaparecido por estar elaborado en una materia perecedera (piel, fibras vegetales, corteza).

Todos estos aspectos nos hacen pensar que efectivamente el varón del hipogeo CCA-3 pudo haberse inhumado con un proyectil alojado en su interior.

\section{AGRADECIMIENTOS}

Los análisis del utillaje lítico y óseo se han realizado en el marco del proyecto de investigación I+D financiado por el Ministerio de Economía y Competitividad: "Aproximación a las primeras comunidades neolíticas del noreste peninsular a través de sus prácticas funerarias" (HAR2011-23149, http://sepulturasneoliticas.blogspot.com.es/2013/03/presentacion-objetivos-de-nuestro.html consulta 8-I-2014), dirigido por J. F. Gibaja Bao. Agradecemos a la Policía Científica dels Mossos d'Esquadra su ayuda en el análisis de la detección de hemoglobina en el geométrico.

\section{BIBLIOGRAFÍA}

Armentano, N.; Esteve, X.; Nociarová, D. y Malgosa, A. 2012: "Taphonomical study of the anthropological remains from Cova Des Pas (Minorca)". Quaternary International 275: 112-119.

Brothwell, D. R. 1987: Desenterrando huesos. Fondo de Cultura Económica. México.

Buc, N. 2011: "Experimental series and use-wear in bone tools". Journal of Archaeological Science 38 (3): 546-557. doi: 10.1016/j.jas.2010.10.009.

Buikstra, J. E. y Ubelaker, D. H. 1994: Standards for data collection from human skeletal remains. Arkansas Archaeological Survey Research Series 44, Arkansas Archaeological Survey. Fayetteville. Arkansas.

Chimenos, E.; Safont, S.; Alesan, A.; Alfonso, J. y Malgosa, A. 1999: "Propuesta de protocolo de valoración de parámetros en Paleodontología". Gaceta Dental 102: 44-52.

DiMaio, V. y DiMaio, D. 2001: Forensic Pathology, Second Edition. CRC Press. Boca Raton.

Duday, H.; Courtaud, P.; Crubezy, E.; Sellier, P. y Tillier A. M. 1990: "L'anthropologie de terrain. Reconnaissance et interprétation des gestes funéraires". Bulletin et Mémoires de la Société d'Anthropologie de Paris 2 (3-4): 29-50.

Gibaja, J. F. 2003: Comunidades Neolíticas del Noreste de la Península ibérica. Una aproximación socio-económica a partir del estudio de la función 
de los útiles líticos. British Archaeological Reports, International Series 1140, Archaeopress. Oxford.

Gibaja, J. F. y Palomo, A. 2004: "Geométricos usados como proyectiles. Implicaciones económicas, sociales e ideológicas en sociedades neolíticas del VI-III milenio Cal BC en el Noreste de la Península Ibérica". Trabajos de Prehistoria 61 (1): 81-97. doi:10.3989/tp.2004.v61.i1.30.

Gijn van, A. 2005: "A functional analysis of some late Mesolithic bone and antler implements from the Dutch coastal zone". En J. Luik, A. Choyke, C. E. Batey y L. Lougas (eds.): From Hooves to Horns, from Mollusc to Mammoth. Manufacture and Use of Bone Artefacts from Prehistoric Times to the Present. Muinasaja Teadus 15, Tallinn Book Printers. Tallinn: 47-66.

González, P.; Martín, A. y Mora, R. 1999: Can Roqueta. Un establiment pagès prehistòric $i$ medieval. Excavacions arqueològiques a Catalunya 16, Departament de Cultura de la Generalitat de Catalunya. Barcelona.

Haglund, W. D. y Sorg, M. H. 2001: Advances in Forensic Taphonomy. Method, Theory and Archaeological Perspectives, CRC Press. Boca Raton.

Hochmeister, M. N.; Budowle, B.; Sparkes, R.; Rudin, O.; Gehrig, C.; Thali, M.; Schmidt, L.; Cordier, A. y Dirnhofer, R. 1999: "Validation studies of an immunochromatographic 1-step test for the forensic identification of human blood". Journal of Forensic Science 44 (3): 597-602.

Isidro, A. y Malgosa, A. 2003: Paleopatología. La enfermedad no escrita. Masson. Barcelona.

Krogman, W. M. e Iscan, Y. M. 1986: The Human Skeleton in Forensic Medicine. Ch. C. Thomas Ed. Springfield. Illinois.

Lyman, R. L. 1994: Vertebrate Taphonomy, Cambridge University Press. Cambridge.

Lovejoy, C. O.; Meindl, R.; Pryzbeck, T. R. y Mensforth, R. P. 1985: "Chronical Metamorphosis of the
Auricular Surface of the Ilium: A New Method for the Determination of Adult Skeletal Age at Death". American Journal of Physical Anthropology 68: 1528.

Martín, A. 2009: "Les sociétés du Néolithique moyen en Catalogne et leur gestion du funéraire". En J. Guilaine (ed.): Sépultures et Sociétés. Du Néolithique à l'Histoire. Editions Errances. París.

Martin, R. y Saller, K. 1957: Lehrbuch des Anthropologie. Ed. G. Fischer. Sttutgart.

Olivier, G. 1960: Practique Anthropologique. Vigot Frères Eds. París.

Pou, R. y Martí, M. 1997: Excavacions arqueològiques a la ronda Sud de Granollers, 1994. La necrópolis del neolític mitjà $i$ les restes romanes del Camí de can Grau (La Roca del Vallès, Vallès Oriental). Els jaciments de Cal Jardiner (Granollers, Vallès Oriental). Excavacions arqueològiques a Catalunya 14, Departament de Cultura, Generalitat de Catalunya. Barcelona.

Pou, R.; Martí, M.; Diaz, J. y Bordas, A. 1994: “Estudio de la necrópolis del grupo Sepulcros de Fosa del yacimiento de 'Bòbila Madurell' (Sant Quirze del Vallès, Barcelona) en el contexto del Neolítico Medio Reciente en Catalunya". En V. M. Oliveira Jorge (ed.): $1 .{ }^{\circ}$ Congresso de Arqueologia Peninsular (Porto 1993): 61-76. Porto.

Roig, J.; Coll, J. M.; Gibaja, J. F.; Chambon, P.; Villar, V.; Ruiz, J.; Terradas, X. y Subirà, M. E. 2010: "La necrópolis de Can Gambús-1 (Sabadell, Barcelona). Nuevos conocimientos sobre las prácticas funerarias durante el Neolítico medio en el Noreste de la Península Ibérica". Trabajos de Prehistoria 67 (1): 59-84. doi: 10.3989/tp.2010.10031.

Ubelaker, D. H. 1989: Human Skeletal Remains. Excavation, Analysis, Interpretation. Taraxacum. Washington. 